\title{
Effects of Shock Waves on Freeway Crash Likelihood
}

\author{
Chris Lee* and Steven Volpatti
}

\author{
Department of Civil and Environmental Engineering, University of Windsor, Windsor, Ontario, N9B 3P4 Canada
}

\begin{abstract}
This study examines how the formation and dissipation of a queue indicated by shock waves affect the likelihood of crash occurrence on freeways. Using one-minute average volume and density data collected from a section of the Gardiner Expressway in Toronto, changes in volume and density 3-10 minutes prior to the time of crash occurrence were observed. Types of shock wave and the shock wave speeds were estimated and related to the frequencies of the crashes where the shock wave existed before they occurred. It was found that typical shock wave types vary in different time periods of day due to different traffic conditions. The comparison with the volume-density data for the non-crash cases using logistic regression models shows that crashes are more likely to occur when the forward shock wave speed is lower. This indicates that slower vehicle progression in near-capacity conditions and slower dissipation of a queue in congested conditions are more likely to cause crashes. The results provide insights into better understanding of how shock waves affect the crash likelihood.
\end{abstract}

Keywords: Crash, freeway, shock wave, real-time, logistic regression.

\section{INTRODUCTION}

Over the past few years, real-time traffic flow data collected from loop detectors have been widely used for monitoring traffic conditions on freeways. More recently, some researchers have proposed to use the same data for detecting crash-prone conditions for proactive crash prevention. In the researchers' work, these detector data have been used for mainly identifying the current traffic states. However, they have been rarely used for detecting the shortterm transition of traffic states. In particular, the formation and dissipation of congestion creates greater variation in speed and potentially increases likelihood of crash occurrence on freeways [1,2].

Most studies on real-time freeway crash likelihood have identified various traffic flow measures using the traffic flow data collected for some time period before a crash occurs. Different types of probabilistic models were developed by relating these traffic flow measures to crash frequencies or crash likelihood. For instance, Oh et al. [3] predicted the real-time likelihood of freeway crashes using the probability density functions that distinguish disruptive traffic conditions from normal traffic conditions. Lee et al. [4] identified real-time crash precursors such as variation in speed and traffic density, and predicted potential for crashes using a log-linear model that accounts for exposures. AbdelAty et al. [5] also identified traffic measures obtained from several detector stations upstream and downstream of the crash site and calculated the probability of crashes using the matched case-control logistic regression model. Golob et al. [6] found a strong association of traffic flow variables collected 30 minutes prior to the crash time with the type of

*Address correspondence to this author at the Department of Civil and Environmental Engineering, University of Windsor, Windsor, Ontario, N9B 3P4 Canada; Tel: + 1-519-2533000; Fax: + 1-519-9713686;

E-mail: cclee@uwindsor.ca crashes. Songchitruksa and Balke [7] considered not only traffic flow variables, but also visibility, lighting and time of day as the real-time predictors for the type of crash using nested logit models. Hourdos et al. [8] observed the empirical relationship between real-time traffic conditions and the crash likelihood using individual vehicle speeds and headways captured by video cameras. Qi et al. [9] developed a crash frequency model using time series and cross sectional measures, and found that real-time traffic flow characteristics, weather, and road geometry are closely related to freeway crash likelihood. Most recently, Son et al. [10] related the individual vehicle data to crash frequencies and found that shorter average distance headway generally increases the crash likelihood.

However, these studies could not capture the effect of the growth and dissipation of a queue on the crash likelihood due to inherent limitations of the selected traffic flow variables. The variables include average speed, volume and occupancy (or density) and their variations over the short time interval prior to crash occurrence. Average volume cannot alone indicate the level of congestion since the same volume can occur at both congested and uncongested conditions. Average speed and density do not indicate whether a queue (if it exists) is forming or dissipating. Although temporal variation in traffic flow such as standard deviation of speed within short time intervals can reflect the queue movement, it cannot show how fast the queue grows or dissipates.

In this regard, shock wave theory can be applied to observing the transition of different traffic states and the speed of such transition. The shock wave speed is conventionally represented by the rate of change in volume and density $[11,12]$. Using this classical theory, the shock wave speed on freeways have been measured and analyzed in the past studies. Messer et al. [13] estimated the speed of shock waves after lane-blocking incidents occurred using the 
combined equations of the kinematic wave model and the Greenshields' macroscopic traffic flow models. Hurdle and Son [14] estimated the shock wave speed using density contour maps that can display the spatial and temporal propagation of the traffic regimes with similar densities. Windover and Cassidy [15] observed the propagation of shock waves using the re-scaled cumulative vehicle arrival curve. While these studies used the aggregated traffic flow data at fixed locations of freeways, Lu and Skabardonis [16] estimated the shock wave speed using individual vehicle trajectories under congested conditions. However, none of these studies considered the association of the shock wave speed with freeway crash likelihood. Also, since the traffic data from loop detectors are more readily available than vehicle trajectories in most freeways, it is more practical to develop the method of detecting the presence and speed of shock waves using the short-term aggregated traffic data.

Thus, the objectives of this study are to determine the type and speed of shock waves using real-time traffic flow data and to evaluate the effects of shock waves on freeway crash likelihood. Following the first section (Introduction), the paper is composed of three subsequent sections. The second section describes the traffic flow data used in the study and typical traffic conditions in the studied freeway. The section also illustrates the method of determining the types of shock waves and estimating the shock wave speed using the traffic flow data. The third section analyzes the association of shock waves with the crash likelihood and explains their effects from empirical perspectives. The last section summarizes the findings and recommends the future studies.

\section{METHODOLOGY}

\section{Description of Data}

This study uses the traffic flow data collected from loop detectors on a section of the westbound Gardiner Expressway in Toronto, Canada. The data include 20-second average speed, volume and occupancy in each lane. There are five loop detector stations that are separated by approximately 500 meters as shown in Fig. (1). There are one entrance ramp from Jameson Avenue and one exit ramp. The entrance ramp is closed for 3 hours from $3 \mathrm{pm}$ to $6 \mathrm{pm}$ during weekdays to avoid congestion in the afternoon peak periods. This section of the freeway was chosen because relatively high number of crashes has occurred in the section compared to the other sections. Also, the traffic becomes severely congested immediately after the entrance ramp is re-opened at $6 \mathrm{pm}$ and it is easier to observe shock waves.

A total of 104 crashes have occurred on this section of the road during weekdays of the 13-month period from January 1998 to January 1999. The crash data were obtained from the freeway incident logs that contain date and time of the detected crash (later verified by a traffic control operator), the nearest loop detector station and the number of blocked lanes after the crash occurrence. Since the police crash reports were not available, the type of crashes was unknown. It was visually observed that the speed at the detector station immediately upstream of the crash site abruptly dropped after the reported time of crash occurrence due to lane blockage. Since it is important to distinguish the pre-crash conditions from the post-crash conditions, actual time of crash occurrence must be accurately estimated. In this study, the time of crash occurrence was assumed to be the time when the speed abruptly dropped. Among 104 crashes, $62 \%$ of crashes have occurred at the station 80 immediately upstream of the exit ramp. The reason for high crash frequency at this location is unknown.

To understand the temporal patterns of traffic conditions on the studied freeway, the volume-density relationships were observed for the following four time periods: 1) morning peak period (6-9 am); 2) off-peak period ( 9 am-3pm and $8-11 \mathrm{pm})$; 3) afternoon peak period when the entrance ramp is closed (3-6 pm); and 4) afternoon peak period when the entrance ramp is opened (6-8 pm). The original 20second average data were converted to 1-minute average data to eliminate large fluctuation of the values. Traffic conditions were observed using the 1-minute volume and density (or occupancy) collected from loop detectors in different time periods.

Traffic conditions were classified into the uncongested and congested regimes based on the density at capacity (or critical density). The uncongested and congested regimes represent the conditions when density is lower and higher, respectively, than the critical density. The capacity was estimated to be 2,300 vehicles/hour/lane, i.e. the maximum observed volume, and the critical density was determined to be 30 vehicles $/ \mathrm{km}$ when the volume reaches the capacity.

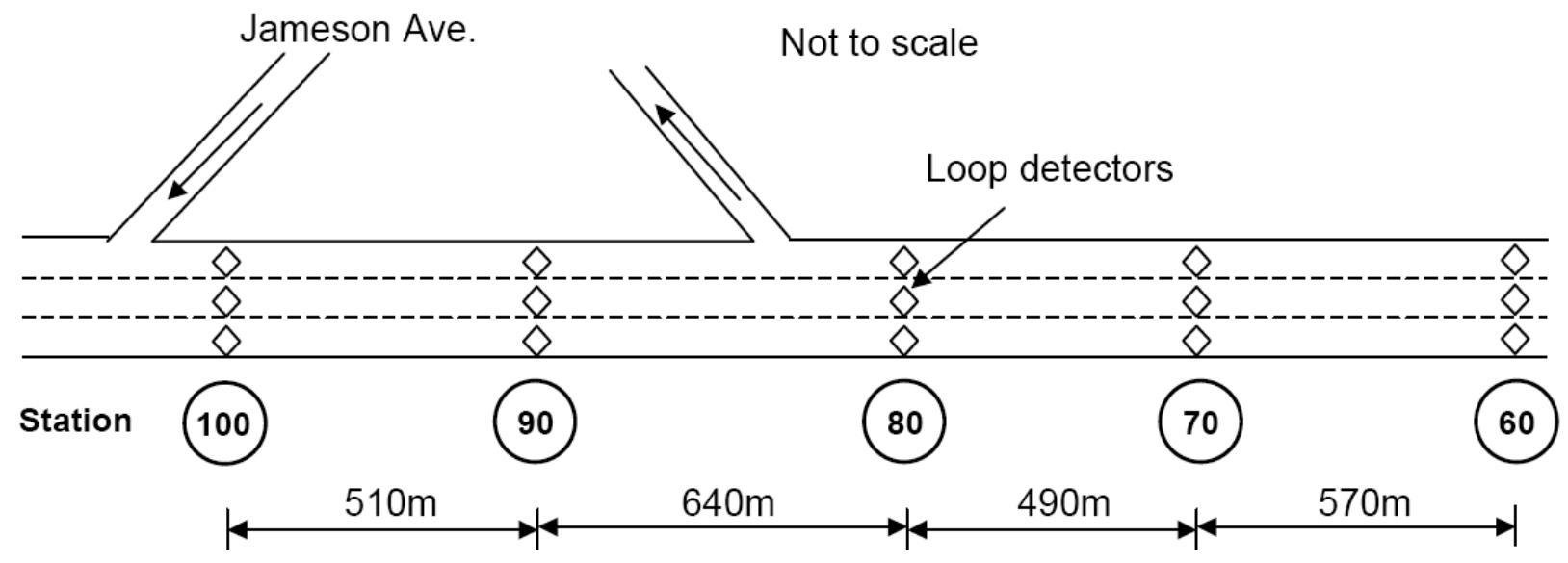

Fig. (1). Location of detectors on westbound Gardiner expressway. 
Fig. (2) shows the volume-density relationships by time of day on several weekdays during the study period. In the morning peak period, volume-density data points generally lie in the uncongested regime as shown in Fig. (2a). In the off-peak period, the data points are scattered over both uncongested and congested regimes as shown in Fig. (2b). In the afternoon peak periods, the points generally lie in the congested regime as shown in Fig. (2c, d). In particular, a greater portion of the data points lies in the congested regime during the period with opened entrance ramp than the period with closed entrance ramp. This indicates that congestion is more severe after the ramp is opened. Clearly typical traffic conditions vary in different time periods.

However, the figures do not display short-term variation in traffic flow and short-lasting queue. The formation and dissipation of a queue can be observed by tracing the change in traffic conditions. This change can be represented by shock waves. The estimation of the shock wave speed is explained in the next section.

\section{Estimation of Shock Wave Speed}

If shock wave exits, the shock wave speed is determined as the change in volume divided by the change in density as

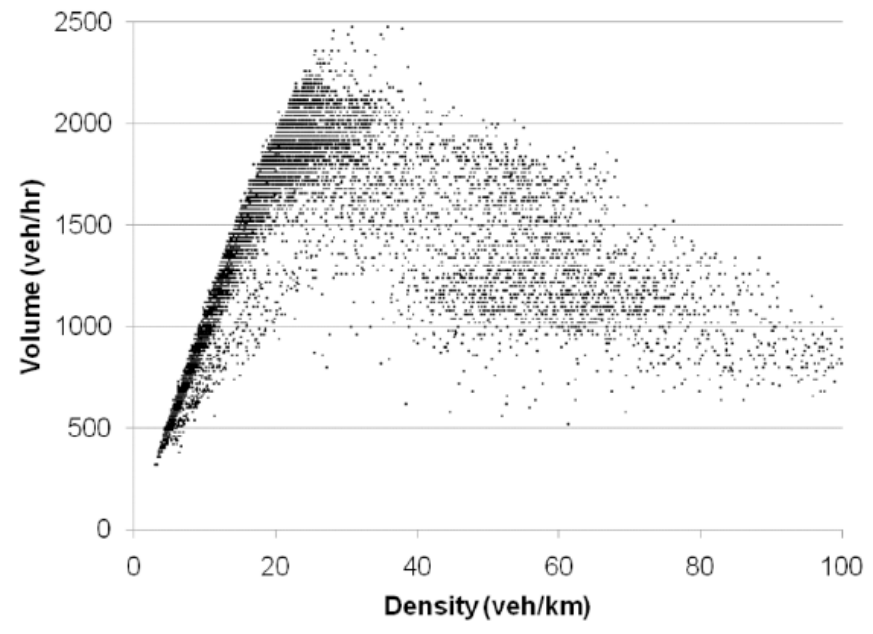

(a) Morning peak period

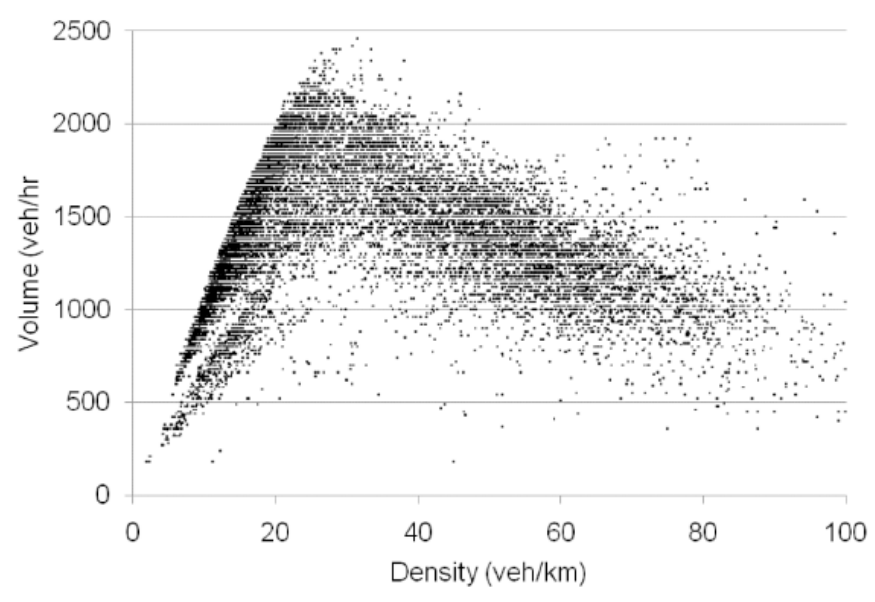

(b) Off peak period

Fig. (2). Volume-density relationship in different time periods. follows:

$\omega_{\mathrm{AB}}=\frac{\Delta q}{\Delta k}=\frac{\mathrm{q}_{\mathrm{A}}-q_{B}}{\mathrm{k}_{\mathrm{A}}-k_{B}}$

where $\omega_{A B}$ is the speed of the shock wave moving from traffic state $A$ to traffic state $B(\mathrm{~km} /$ hour $), q_{A}$ and $q_{B}$ are volumes at traffic states $A$ and $B$, respectively (vehicles/hour), and $\mathrm{k}_{\mathrm{A}}$ and $\mathrm{k}_{\mathrm{B}}$ are densities at traffic states $\mathrm{A}$ and $\mathrm{B}$, respectively (vehicles $/ \mathrm{km}$ ).

In this study, the shock wave speed was estimated using the volume and density during 10 minutes prior to the estimated time of crash occurrence. If the 1-minute average volume and density consistently increase or decrease during the 10-minute period, it is possible that the shock wave contributed to crash occurrence. The shock wave speed was estimated by measuring the slope of a line that best fits to the observed data points. The line can be fit to the data more objectively using the least-square linear regression.

In some cases, it was observed that although the volume and density did not consistently increase or decrease during the entire 10-minute period, there were two distinct clusters of points. These clusters were assumed to be the two

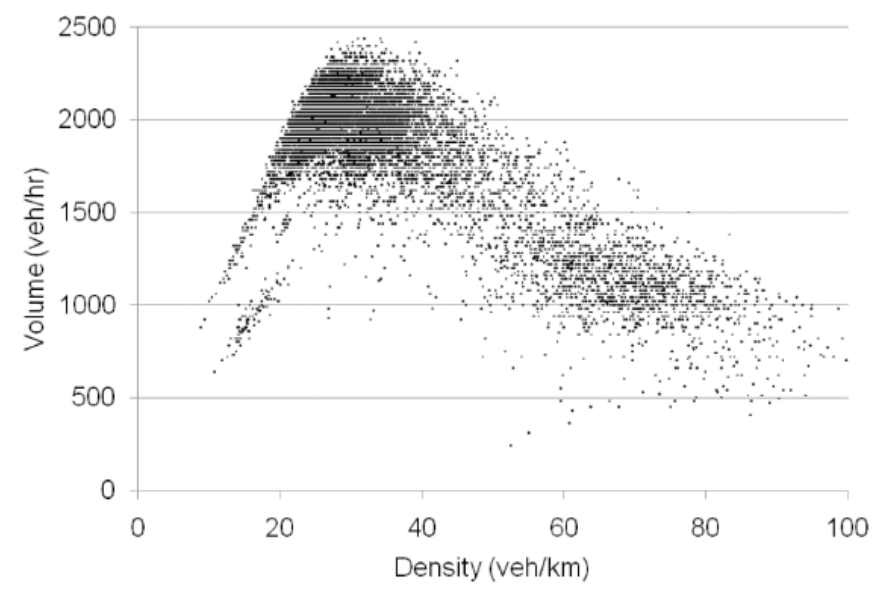

(c) Afternoon peak period when the entrance ramp is closed (3-6 pm)

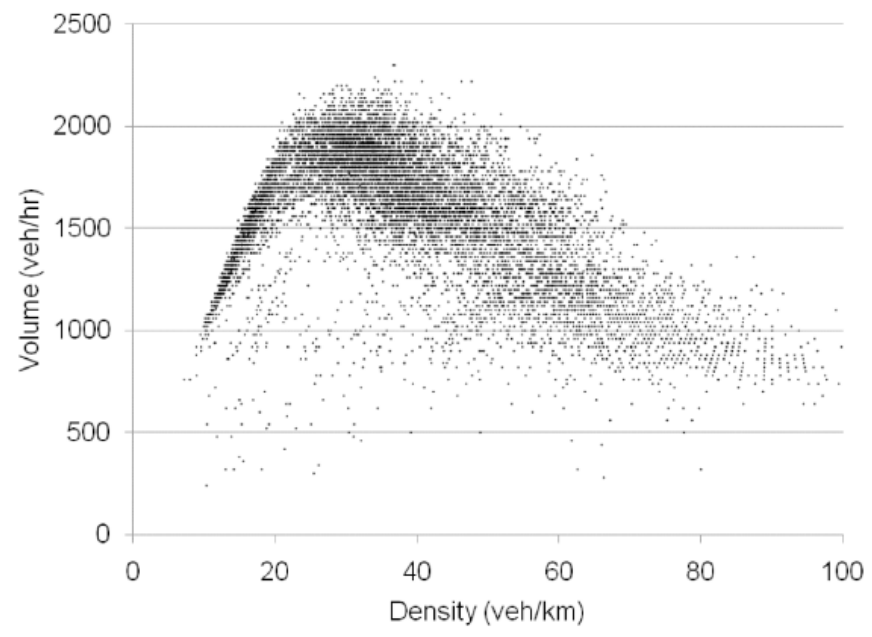

(d) Afternoon peak period when the entrance ramp is open (6-8 pm) 
different steady-state traffic states. Thus, the average volume and density were calculated for each cluster as a representative point and the slope of a line connecting these representative points was measured.

However, shock waves could not be measured for 45 crashes among 104 crashes due to no specific pattern of changes in volume and density. This implies that the shock wave did not exist prior to the occurrence of some crashes. Thus, the scope of this analysis will be limited to the crashes where shock waves existed before the crashes occurred.

In this study, the shock wave was classified in terms of the following categories:

1. Direction of shock wave movement: forward or backward shock wave. Forward shock wave moves in the same direction as traffic flow whereas backward shock wave moves in the opposite direction to traffic flow.

2. Growth or dissipation of a queue: forming or recovery shock wave. Forming shock wave occurs as the density increases whereas recovery shock wave occurs as the density decreases.

3. Traffic state: changes in volume and density within the same traffic states (uncongested or congested regime) or between two different traffic states (from the uncongested regime to the congested regime or

\section{Type 1: Forward Forming}

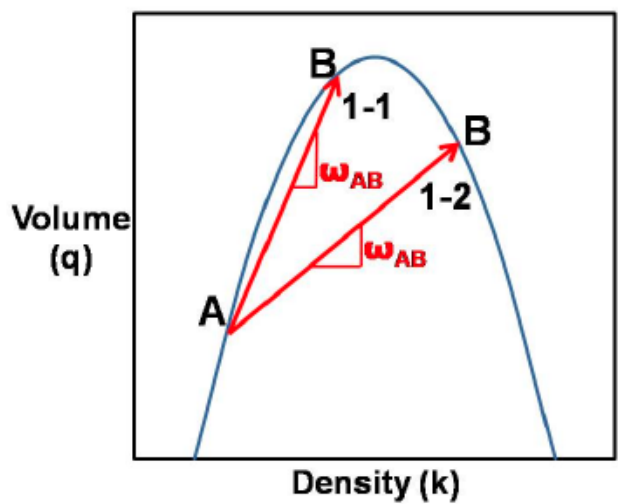

Type 3: Backward Forming

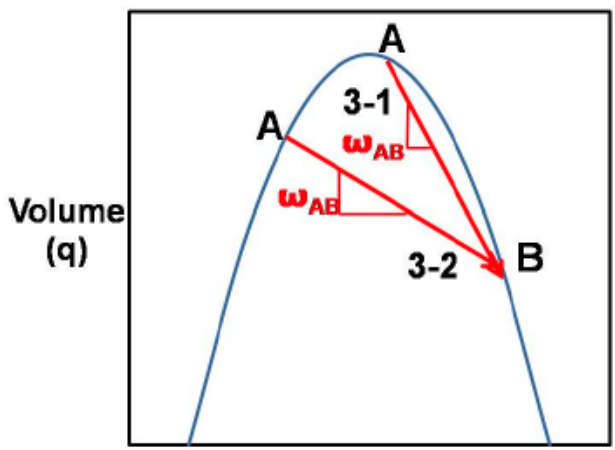

Density (k) vice versa).

The following eight shock wave types were defined based on the above categories:

Type 1-1: Forward forming shock wave within uncongested regime

Type 1-2: Forward forming shock wave from uncongested regime to congested regime

Type 2-1: Forward recovery shock wave within uncongested regime

Type 2-2: Forward recovery shock wave from congested regime to uncongested regime

Type 3-1: Backward forming shock wave within congested regime

Type 3-2: Backward forming shock wave from uncongested regime to congested regime

Type 4-1: Backward recovery shock wave within congested regime

Type 4-2: Backward recovery shock wave from congested regime to uncongested regime

The eight shock wave types are also shown in Fig. (3). As shown in the figure, the shock wave speed $\left(\omega_{\mathrm{AB}}\right)$ represents the slope of the line $\mathrm{AB}$ in a volume-density

\section{Type 2: Forward Recovery}

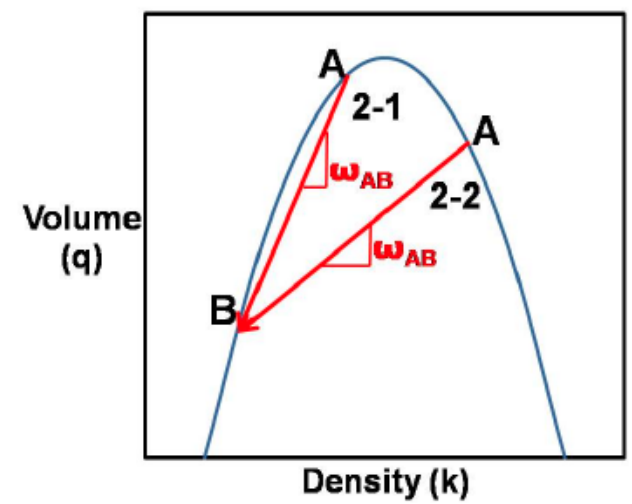

Type 4: Backward Recovery

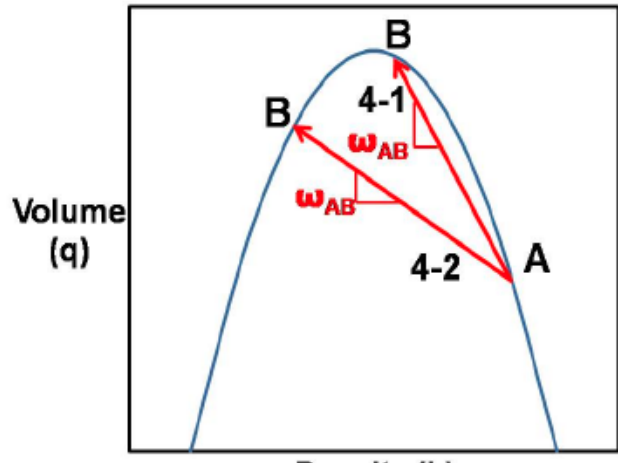

Density (k)

Fig. (3). Types of shock wave. 
curve. The shock wave speeds with positive and negative slope represent forward and backward shock wave, respectively.

Table 1 shows frequencies of crashes by shock wave type for different time periods. Forward shock wave (Types 1 and 2 ) is the dominant shock wave type associated with crashes in the morning peak and off-peak periods. On the other hand, both forward shock wave (Types 1 and 2) and backward shock wave (Types 3 and 4) are almost equally associated with crashes in the afternoon peak period. It was also observed that crashes are associated with both forming and recovery shock waves for backward shock waves but nearly all crashes are associated with forming shock waves for forward shock waves. This implies that crashes are more likely to occur when a queue grows in the uncongested regime and a queue grows or dissipates in the congested regime.

As noted earlier, shock waves could not be identified for some crashes due to no consistent patterns of volume and density changes for the entire 10-minute period prior to the crash time. However, it is possible that shock waves only existed for a time period shorter than 10 minutes. For this reason, traffic conditions for the time period shorter than 10 minutes prior to the crash time (called "short-term interval") were observed.

For instance, shock waves were determined during 3 4 minutes prior to the crash time as shown in Fig. (4). The plots on the left of the figure show the conditions for the 10minute intervals and indicate which traffic regime (uncongested or congested) the current traffic state belongs to. The short-term intervals were selected based on the authors' subjective judgment such that more consistent patterns can be observed during the selected interval. The figure demonstrates that the change in the short-term intervals better captures the patterns of volume and density changes immediately before the crash time than the change in 10-minute intervals. In Fig. (4a, b), although the slope of the regression line is positive during 10 minutes, volume and density did not consistently increase or decrease over the entire 10-minute interval. However, the data points during 3 minutes prior to the crash time clearly show consistent pattern of change in which both volume and density increase (forward forming shock wave) or decrease (forward recovery shock wave). Similarly, the data points during 4 minutes prior to the crash time in the afternoon peak periods show negative slope of the regression line and consistent pattern of change in which volume increases while density decreases (backward recovery shock wave) or volume decreases while density increases (backward forming shock wave) as shown in Fig. (4c, d).

As a result, shock waves could be determined for 72 crashes which are more than 59 crashes initially determined for the 10-minute interval prior to the crash time. Frequencies of crashes by shock wave type for the shortterm intervals are also shown in Table 1. It was found that the shock wave types for some crashes were different between the 10-minute and short-term intervals. It should be noted that a substantial portion of the shock waves previously classified as forming shock waves for the 10minute interval was re-classified as recovery shock waves for the short- term interval. For this reason, the distributions of crashes by shock wave type were different between 10minute and short-term intervals. The estimated shock wave speeds by shock wave type for the both time intervals are shown in Table 2. Although the backward shock wave speed is conventionally expressed in a negative value due to opposite signs of changes in volume and density (refer to Equation 1), an absolute value of the shock wave speed was shown in the table. Given that a crash is more likely to be affected by the short-term change in traffic states than the long-term change, the shock waves estimated for the shortterm intervals were used for the analysis in the next section.

\section{RESULTS AND DISCUSSION}

To identify the effects of shock waves on crashes, shock waves were also estimated for the normal traffic conditions when crashes did not occur. To control for the effects of road geometric, weather and travel demand, 20 -second average traffic flow data were obtained from the same detector station at the same time of day under the same weather condition as the crash cases but on a different day. Day of week was not controlled due to an insufficient data. These

Table 1. Frequency of Crashes for Each Shock Wave Type in Different Times of Day

\begin{tabular}{|c|c|c|c|c|c|c|c|c|}
\hline TYPE $1-2$ & 0 & 0 & 1 & 1 & 1 & 1 & 2 & 4 \\
\hline TYPE 2-1 & 2 & 8 & 0 & 2 & 0 & 4 & 0 & 1 \\
\hline TYPE 3-1 & 0 & 0 & 0 & 0 & 2 & 1 & 4 & 4 \\
\hline TYPE 3-2 & 0 & 0 & 0 & 0 & 0 & 0 & 0 & 0 \\
\hline TYPE 4-1 & 0 & 0 & 0 & 2 & 1 & 1 & 0 & 6 \\
\hline TYPE 4-2 & 0 & 0 & 0 & 0 & 5 & 6 & 2 & 2 \\
\hline
\end{tabular}



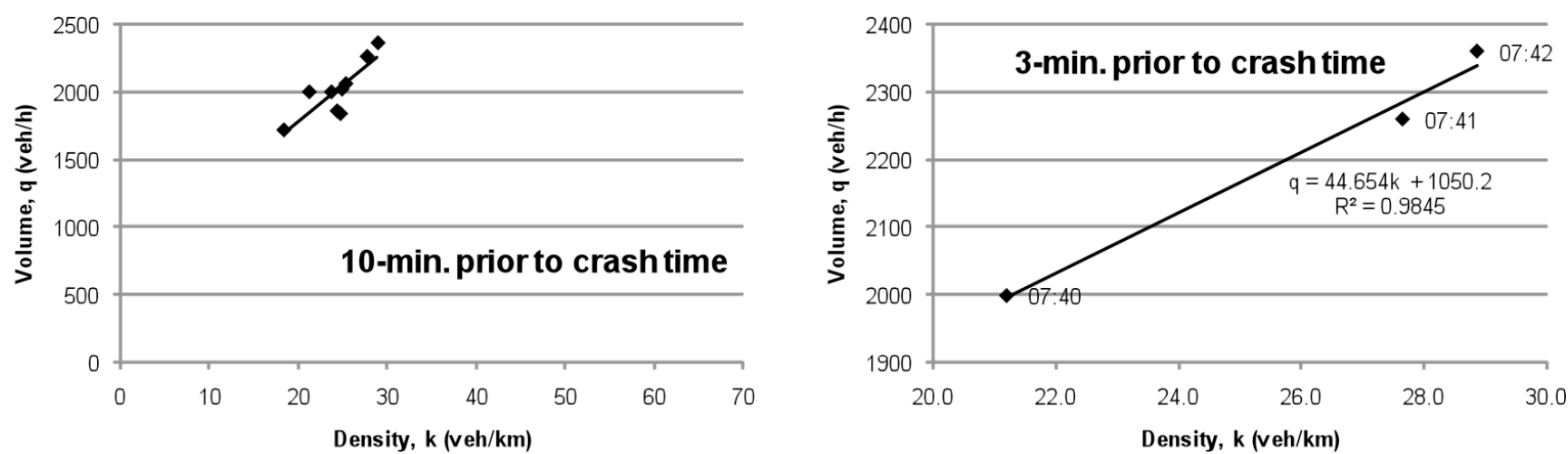

(a) Forward forming shock wave (morning peak period)
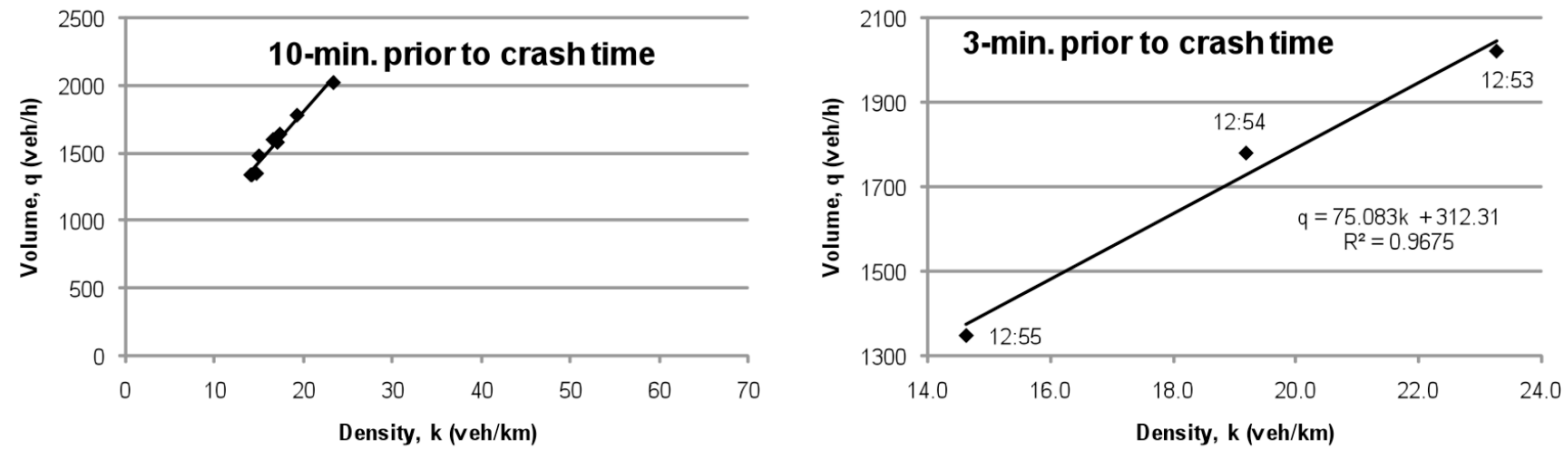

(b) Forward recovery shock wave (Off-peak period)
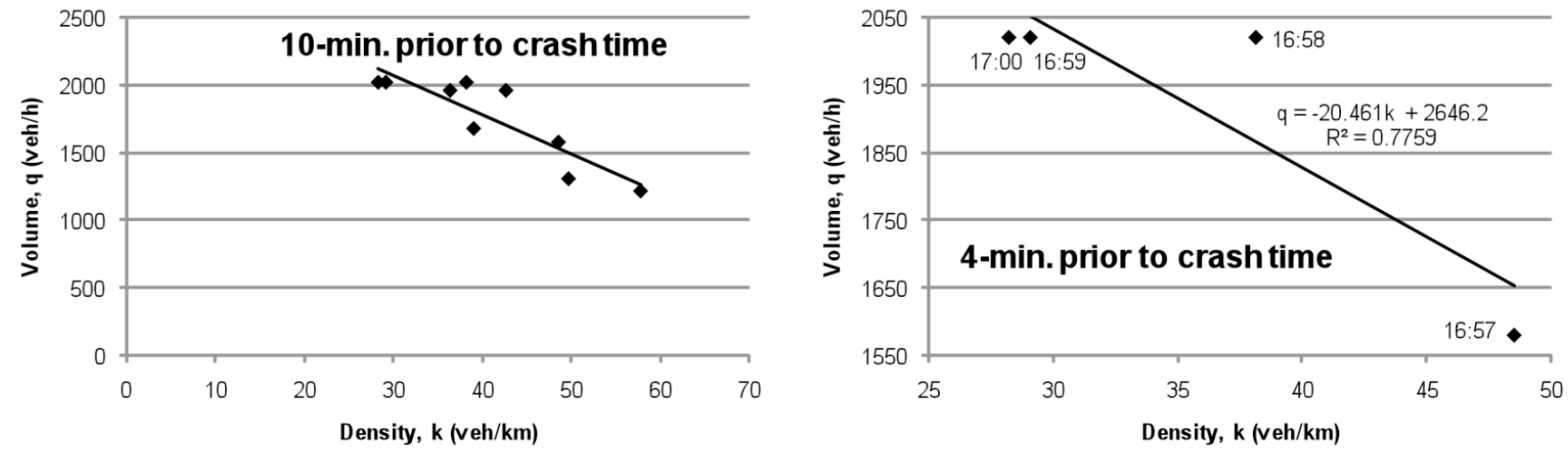

(c) Backward recovery shock wave (afternoon peak period with closed entrance ramp)


(d) Backward forming shock wave (afternoon peak period when open entrance ramp)

Fig. (4). Estimation of shock wave. 
Table 2. Average Shock Wave Speed for Each Shock Wave Type in Different Times of Day for Crash Case (km/h)

\begin{tabular}{|c|c|c|c|c|c|c|c|c|}
\hline Shock Wave Type & 10 Min. & Short-Term & 10 Min. & Short-Term & 10 Min. & Short-Term & 10 Min. & Short-Term \\
\hline TYPE 1-2 & & & 2.27 & 1.67 & 7.80 & 19.80 & 28.22 & 30.92 \\
\hline TYPE 2-1 & 57.71 & 57.66 & & 55.16 & & 55.14 & & 33.19 \\
\hline TYPE 3-1 & & & & & 21.72 & 25.90 & 18.30 & 19.21 \\
\hline TYPE 3-2 & & & & & & & & \\
\hline TYPE 4-1 & & & & 28.11 & 29.00 & 54.49 & & 20.05 \\
\hline TYPE 4-2 & & & & & 19.94 & 16.07 & 14.40 & 14.40 \\
\hline
\end{tabular}

Table 3. Frequency of Non-Crash Cases for Each Shock Wave Type in Different Times of Day

\begin{tabular}{|c|c|c|c|c|c|c|c|c|}
\hline \multirow{2}{*}{ Shock Wave Type } & \multicolumn{2}{|c|}{ AM Peak } & \multicolumn{2}{c|}{ Off Peak } & \multicolumn{2}{c|}{ PM Peak (Ramp Closed) } & \multicolumn{2}{c|}{ PM Peak (Ramp Opened) } \\
\cline { 2 - 9 } & $\mathbf{1 0}$ Min. & Short-Term & $\mathbf{1 0}$ Min. & Short-Term & $\mathbf{1 0}$ Min. & Short-Term & $\mathbf{1 0}$ Min. & Short-Term \\
\hline \hline TYPE 1-1 & 11 & 9 & 10 & 11 & 8 & 10 & 2 \\
\hline TYPE 1-2 & 0 & 0 & 0 & 0 & 2 & 3 & 3 \\
\hline TYPE 2-1 & 9 & 11 & 4 & 3 & 4 & 3 & 0 \\
\hline TYPE 2-2 & 0 & 0 & 0 & 0 & 1 & 3 & 3 \\
\hline TYPE 3-1 & 0 & 0 & 1 & 2 & 2 & 3 & 3 \\
\hline TYPE 3-2 & 0 & 0 & 0 & 0 & 0 & 1 & 3 \\
\hline TYPE 4-1 & 0 & 1 & 0 & 1 & 1 & 1 & 3 \\
\hline TYPE 4-2 & 0 & 0 & 0 & 0 & 0 & 0 & 1 \\
\hline Total Crash & 20 & 21 & 15 & 17 & 18 & 21 & 17 \\
\hline
\end{tabular}

Table 4. Average Shock Wave Speed for Each Shock Wave Type in Different Times of Day for Non-Crash Case (km/h)

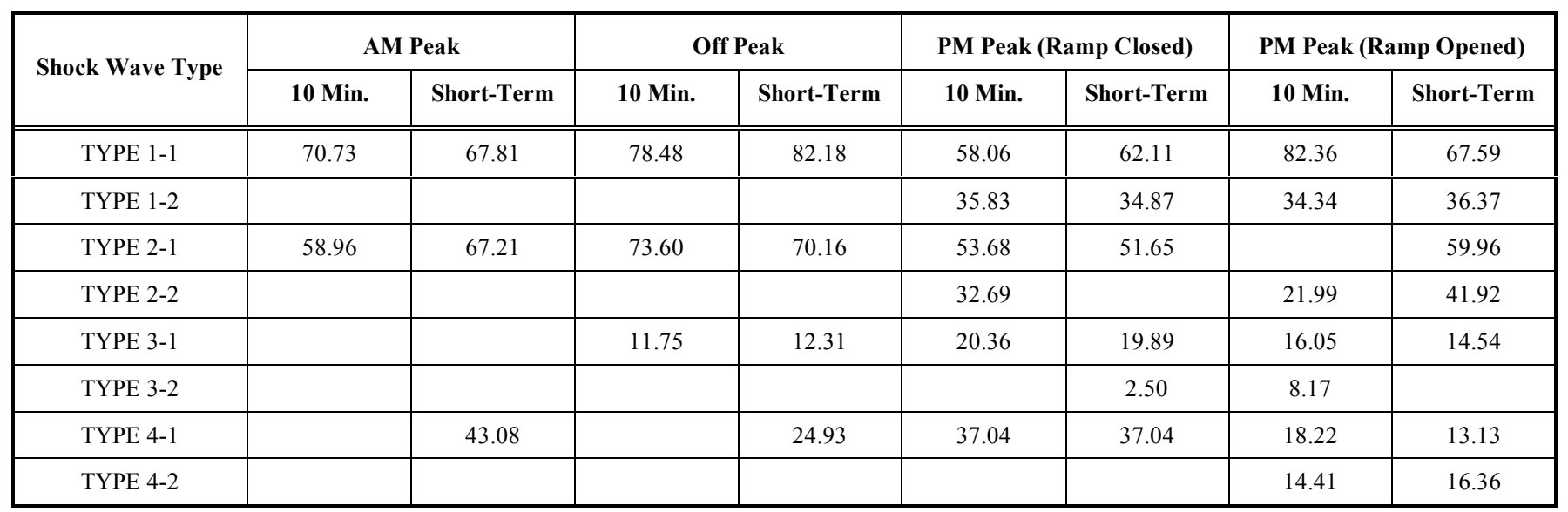

data are called the "non-crash cases". It was assumed that crashes did not occur in these cases due to lower contribution by shock waves to the crash occurrence although shock waves existed. Shock waves were determined in the same manner as the crash cases as illustrated in the previous section.
Frequencies of the non-crash cases by shock wave type for the four time periods are shown in Table 3. Similar to the crash cases, shock waves were determined for the 10-minute and short-term intervals. Shock waves for a total of 78 noncrash cases were determined. The estimated shock wave speeds by shock wave type are shown in Table 4. 


\section{Forward Shock Wave}

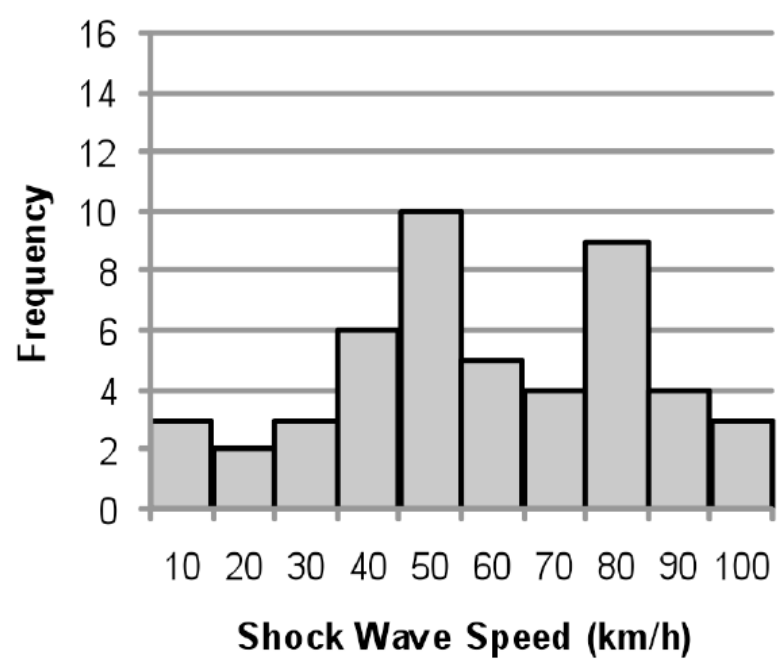

Backward Shock Wave

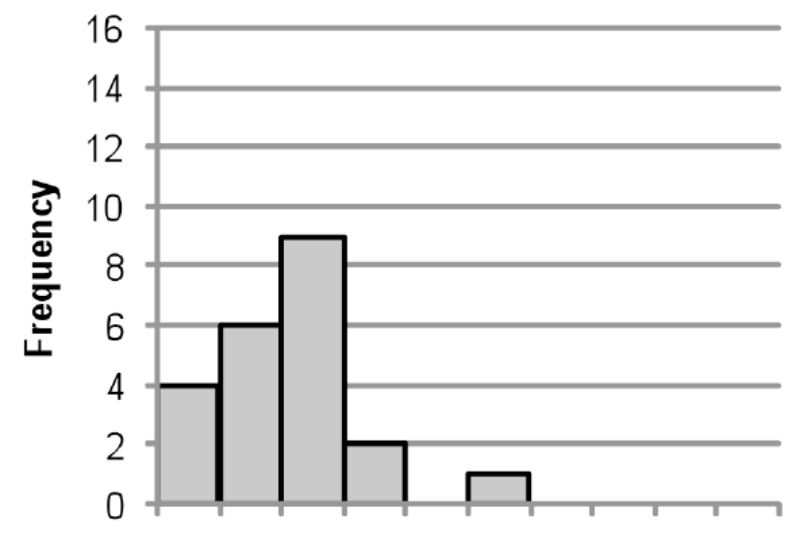

$102030405060 \quad 70 \quad 8090100$

Shock Wave Speed (km/h)

(a) Crash case
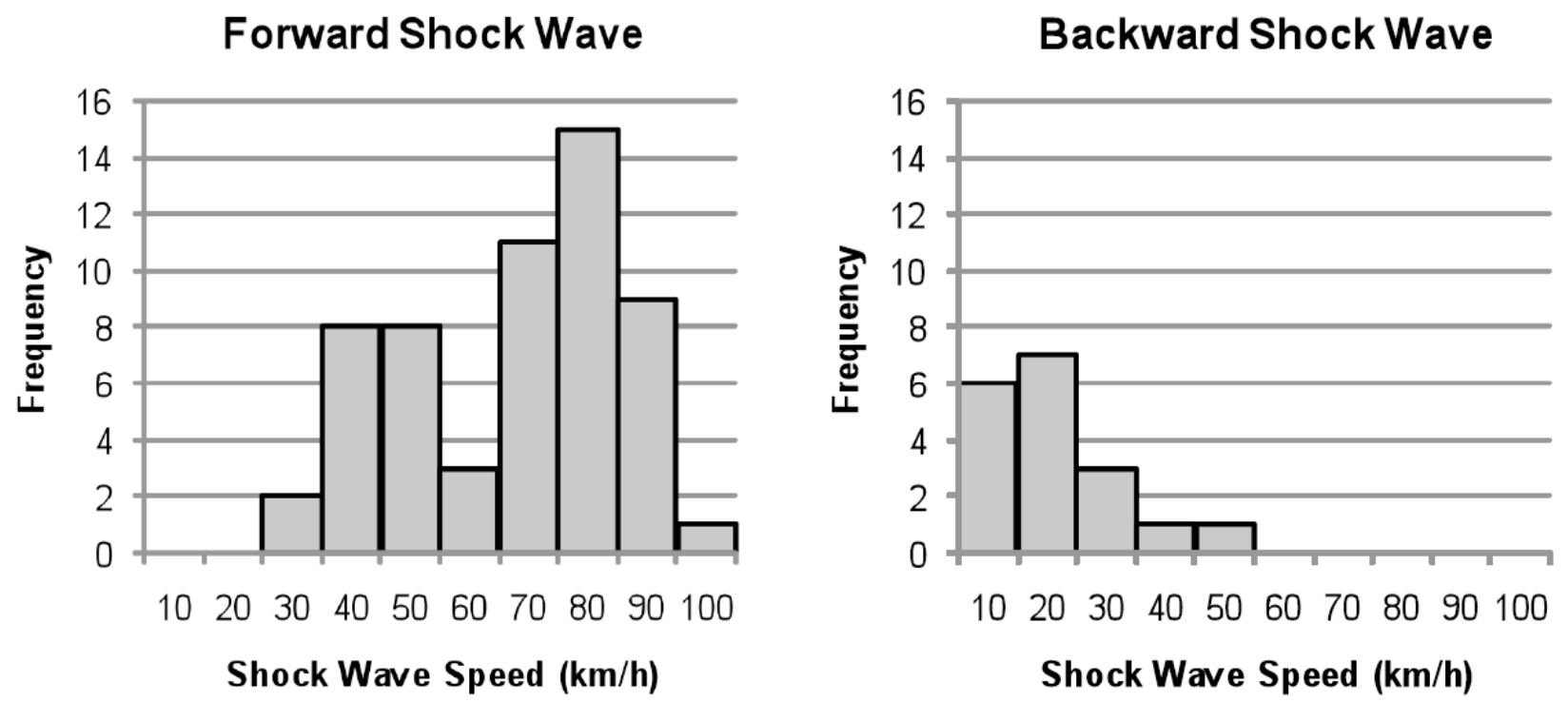

(b) Non-crash case

Fig. (5). Comparison of shock wave speeds by direction of movements for crash and non-crash cases.

Due to the limited amount of data, the crash and noncrash cases were compared based on the distributions of the forward and backward shock waves instead of the 8 shock wave types as shown in Fig. (5). It was found that the average forward shock wave speed was lower for the crash cases than the non-crash cases $(54.46 \mathrm{~km} / \mathrm{h}$ vs $64.72 \mathrm{~km} / \mathrm{h})$ but the average backward shock wave speed was higher for crash cases than the non-crash cases $(21.75 \mathrm{~km} / \mathrm{h} v s 17.64$ $\mathrm{km} / \mathrm{h})$.

However, the difference in the shock wave speed between the crash and non-crash cases was statistically significant at a $95 \%$ confidence interval only for the forward shock wave $(\mathrm{t}$-statistics $=2.27, p$-value $=0.03)$, but not for the backward shock wave (t-statistics $=1.18, p$-value $=$ 0.24). To assess the impact of the shock wave speed on the crash likelihood, the binary logistic regression model was developed. The model predicts the probability of occurrence of a crash based on the shock wave speed (x) as described in the following expression:

$\ln \frac{P(Y=i)}{1-P(Y=i)}=\alpha+\beta x$

where $\mathrm{P}(\mathrm{Y}=\mathrm{i})$ is the probability of occurrence of a crash $(\mathrm{i}=$ 1 for crash and $i=0$ for non-crash), $\alpha$ is the constant, and $\beta$ is the coefficient. The left side of Equation (2) denotes the ratio of the probability of crash $(\mathrm{Y}=1)$ to the probability of 
non-crash $(\mathrm{Y}=0)$. This ratio is also called the odds of crash to non-crash. The odds of crash for forward shock waves is described as follows:

$\ln \left(\frac{P(Y=1)}{P(Y=0)}\right)=1.0119-0.197 x$

The shock wave speed is statistically significant at a $95 \%$ confidence interval ( $p$-value $=0.025)$. The negative coefficient of the shock wave speed implies that lower forward shock wave speed increases the odds of crash. This indicates that a crash is more likely to occur when the forward shock wave propagates slowly. The reason can be explained as follows.

Lower forward shock wave usually occurs near the boundary between the uncongested and congested regimes. It can be verified from a volume-density curve in Fig. (3) that the rate of change in volume for a given change in density is much lower near the capacity than the rate of change in freeflow conditions (low volume and low density). In other words, traffic state radically changes from uncongested conditions to congested or near-capacity conditions. Thus, vehicles are more likely to encounter slow-moving lead vehicles ahead and this will result in higher chance of crashes. Lower forward shock wave also occurs when a queue slowly dissipates in congested conditions. Slower dissipation of a queue increases the duration of congestion and thereby increases chance of abrupt speed transition between congested and uncongested conditions. This finding is consistent with Abdel-Aty et al. [17] which found that faster removal of congestion can effectively reduce crash risk by preventing the growth of a queue.

However, the backward shock wave speed was not statistically significant in the logistic regression model. This result contradicts the priori expectation that that higher backward shock wave speed increases the odds of crash. This is because as a queue grows faster in the opposite direction of traffic flow, the drivers upstream of a queue are more likely to unexpectedly encounter the tail of a queue. Consequently, the drivers' potential failure of reducing speed will result in a crash. Insignificant effect of the backward shock wave is partially supported by the fact that relatively low number of crashes occurred at the locations where backward shock waves formed more frequently. More specifically, although a queue occasionally formed immediately upstream of the entrance ramp after the ramp is opened, fewer crashes occurred at these locations compared to the locations further upstream of the entrance ramp. Another reason for the insignificant effect is relatively fewer samples of the backward shock wave compared to the forward shock wave.

\section{CONCLUSIONS AND RECOMMENDATIONS}

This study investigates the effects of shock waves on the likelihood of crash occurrence on freeways. The types of shock waves and the shock wave speeds were estimated by tracing the changes in the 1-minute average volume and density data 3-10 minutes before a crash occurred if the shock wave existed. Shock waves were compared between the crash and non-crash cases to evaluate the association of shock waves with the crash likelihood. The main findings of the analysis are summarized as follows:

1. Typical shock wave types vary in different time periods of day. Dominant shock wave types are determined by the typical traffic conditions during the time period. If shock waves exist, crashes tend to occur more frequently by dominant shock wave type in each time period.

2. Lower forward shock wave speed increases the crash likelihood. This implies that slower vehicle progression in near-capacity conditions and slower dissipation of a queue in congested conditions are more likely to cause crashes on freeways.

The findings in this study also apply to any location regardless of the number of crashes. The similar results were also found in the eastbound freeway section where relatively lower number of crashes occurred compared to the westbound freeway section [18].

Based on the findings, it is recommended that timevarying volume and density are monitored in real time to detect high crash-prone traffic conditions. When lower forward shock wave speed (classified according to the prespecified threshold value) is detected under the congested conditions, it is important to facilitate removal of congestion by increasing the speed downstream of the bottleneck. These will mitigate the adverse effects of shock waves on high traffic flow turbulence and furthermore the crash likelihood. However, the discussion of specific crash countermeasures is beyond the scope of this study.

In future studies, the effects of the backward shock waves need to be further investigated using additional traffic flow and crash data. This will help better understand how the direction of shock wave movement affects the crash likelihood. It is also recommended that systematic and automated methods of determining the time period and estimating the shock wave speed are developed.

\section{ACKNOWLEDGEMENT}

This work was supported by the Natural Sciences and Engineering Research Council (NSERC), Canada.

\section{REFERENCES}

[1] B. S. Kerner, "The Physics of Traffic: Empirical Freeway Pattern Features, Engineering Applications, and Theory”. Springer, 2004.

[2] E. Hauer, "Accidents, overtaking and speed control", Accident Analysis and Prevention, vol. 3, pp. 1-13, 1971.

[3] C. Oh, J. Oh, S. Ritchie, and M. Chang, "Real-time estimation of freeway accident likelihood", in $80^{\text {th }}$ Transportation Research Board Annual Meeting, 2001.

[4] C. Lee, F. Saccomanno, and B. Hellinga, "Analysis of crash precursors on instrumented freeways", Transportation Research Record, 1748, pp. 1-8, 2002.

[5] M. Abdel-Aty, N. Uddin, F. Abdalla, and A. Pande, "Predicting freeway crashes based on loop detector data using matched casecontrol logistic regression", Transportation Research Record, 1897, pp. 88-95, 2004.

[6] T. F. Golob, W. W. Recker, and V. M. Alvarez, "Freeway safety as a function of traffic flow", Accident Analysis and Prevention, vol. 36, no. 6, pp. 933-946, 2004.

[7] P. Songchitruksa, and K. N. Balke, "Assessing weather, environment, and loop data for real-time freeway incident prediction", Transportation Research Record 1959, pp. 105-113, 2006. 
[8] J. N. Hourdos, V. Garg, P. G. Michalopoulos, and G. Davis, "Realtime detection of crash-prone conditions at freeway high-crash locations", Transportation Research Record 1968, pp. 83-91, 2006.

[9] Y. Qi, B. L. Smith, and J. Guo, "Freeway accident likelihood prediction using a panel data analysis approach", Journal of Transportation Engineering, vol. 133, no. 3, pp. 149-156, 2007.

[10] H. Son, Y.-J. Kweon, and B. Park, "Development of crash prediction models with individual vehicle data", in 88th Transportation Research Board Annual Meeting, 2009.

[11] P. I. Richards, "Shock waves on the highway", Operations Research, vol. 4, no. 1, pp. 42-51, 1956.

[12] M. H. Lighthill and G. B. Whitham, "On kinematic waves II: A theory of traffic flow on long crowded roads", Proceedings of the Royal Society, Series A, vol. 229, pp. 317-345, 1957.

[13] C. J. Messer, C. L. Dudek, and J. D. Friebele, "Method for predicting travel time and other operational measures in real-time during freeway incident conditions", Transportation Research Record, 567, pp. 1-16, 1976.

[14] V. F. Hurdle, and B. Son, "Road test of a freeway model", Transportation Research, A 34, pp. 537-564, 2000.

[15] J. R., Windover and M. J. Cassidy, "Some observed details of freeway traffic evolution", Transportation Research A 35, pp. 881894, 2001.

[16] X. Y. Lu, and A. Skabardonis, "Freeway traffic shockwave analysis: Exploring NGSIM trajectory data", in 86th Transportation Research Board Annual Meeting, 2007.

[17] M. Abdel Aty, J. Dilmore and L. Hsia, Applying variable speed limits and the potential for crash mitigation, Transportation Research Record, 1953, pp. 21-30, 2006.

[18] S. Volpatti. "Rate of growth and dissipation of a queue on freeways and its effects on crash likelihood", M.A.Sc. thesis, University of Windsor, Windsor, ON, Canada, 2009.

Received: December 9, 2009

Revised: December 24, 2009

Accepted: December 25, 2009

(C) Lee and Volpatti; Licensee Bentham Open.

This is an open access article licensed under the terms of the Creative Commons Attribution Non-Commercial License (http://creativecommons.org/licenses/by-nc/

3.0/) which permits unrestricted, non-commercial use, distribution and reproduction in any medium, provided the work is properly cited. 